\title{
Chemically Controlled Bending of Compositionally Anisotropic Microcylinders**
}

\author{
Sampa Saha, Davor Copic, Srijanani Bhaskar, Nicholas Clay, Alessandro Donini, A. John Hart, \\ and Joerg Lahann*
}

Soft materials that can undergo mechanical actuation in response to external stimuli, ${ }^{[1-7]}$ such as changes in temperature, light, $\mathrm{pH}$ value, or ionic strength, have attracted increasing attention because of their potential use as thinfilm actuators, ${ }^{[8-10]}$ smart sutures, ${ }^{[11,12]}$ and soft robots. ${ }^{[1,14]}$ These materials typically require specialty polymers, such as shape-memory polymers ${ }^{[15]}$ or use macroscopically layered films. ${ }^{[16,17]}$ In layered films, the anisotropic distribution of two polymers, or a polymer and a metal, is essential. This creates a mismatch in mechanical properties that gives rise to a defined bending. In principle, this concept is not limited to macroscopic multilayer films, but can be achieved with colloidal materials, as long as the required anisotropy can be realized and different parts of the colloidal object will respond differently to the external stimulus. In recent years, compositionally anisotropic micro- and nanoparticles have been devised using a range of different synthesis methods including microfluidic and lithographic techniques, ${ }^{[18]}$ particle replication in low surface energy templates, ${ }^{[19]}$ selective crosslinking of polybutadiene segments in terpolymers, ${ }^{[20]}$ lithographic patterning of microspheres, ${ }^{[21]}$ electrochemical ${ }^{[22]}$ and photochemical $^{[23]}$ reduction, templating of porous membranes ${ }^{[24,25]}$ and nanotubes, ${ }^{[26]}$ surfactant aided growth, ${ }^{[27]}$ graft polymerization, ${ }^{[28-30]}$ and processes based on controlled surface nucleation. ${ }^{[31]}$ Alternatively, electrohydrodynamic co-jetting is a method to prepare particles and fibers with multiple compartments by transferring fluids through a set of capillaries that can process dissimilar materials. ${ }^{[32]}$ In the past, electrohydrodynamic co-jetting has resulted in particles with multiple compartments that contain different polymer blends,

[*] Dr. S. Saha, N. Clay, A. Donini, Prof. J. Lahann

Department of Chemical Engineering

University of Michigan, Ann Arbor, MI 48109 (USA)

E-mail: lahann@umich.edu

Dr. S. Bhaskar, Prof. J. Lahann

Macromolecular Science and Engineering Program

University of Michigan, Ann Arbor, MI 48109 (USA)

D. Copic, Prof. A. J. Hart

Department of Mechanical Engineering

University of Michigan (USA)

[**: We thank the American Cancer Society (RSG-08-284-01-CDD) for financial support and acknowledge funding from the Multidisciplinary University Research Initiative of the Department of Defense and the Army Research Office (W911NF-10-1-0518). Additionally, D.C. acknowledges the Rackham Merit Fellowship at the University of Michigan, and D.C. and A.J.H. acknowledge the Air Force Office of Scientific Research (11-NA-018).

Supporting information for this article is available on the WWW under http://dx.doi.org/10.1002/anie.201105387. dyes, low-molecular weight additives, reactive molecules and even inorganic nanoparticles. ${ }^{[33,34]}$ If a reactive additive, such as a functionalized polymer, is added to one of the compartments, selective surface modification is possible and can result in spatially controlled immobilization of proteins or peptides. ${ }^{[35-37]}$

Because different compartments can be loaded with dissimilar materials, entirely new sets of functions can arise from unique synergistic effects, ${ }^{[36]}$ not just from the addition of the properties of the individual compartments.

Herein, we report a new type of compositionally anisotropic microcylinders, where defined compartments within the same microcylinder undergo differential expansion due to the site-selective growth of a surface layer. The asymmetric expansion creates surface stresses resulting in significant and controllable bending of the microcylinders, which depends on the particle geometry and the architecture of the surface layers. Using finite element simulations, we verify the observed bending trends and derive a family of performance curves that predict a wide-range tunability of the actuation stroke based on the cylinder geometry and the amount of swelling.

The microcylinders are fabricated based on electrohydrodynamic co-jetting followed by microsectioning. ${ }^{[34]}$ In brief, an electric field is applied to a compound droplet comprising two or more polymer solutions generated by laminar flow from a side-by-side arrangement of capillary needles. ${ }^{[32,38]}$ We have previously demonstrated the synthesis of particles ${ }^{[35,39]}$ and fibers ${ }^{[33]}$ from chloroform-based solutions of lactic acid polymers. In the case of fibers, high viscosities, combined with high solvent volatility and low charge-to-volume ratios, can result in an extremely linear and controlled jet migration without the bending and whipping instabilities commonly observed in charged jets. This situation enables the production of multicompartmental microfibers, which not only exhibit monodispersity with respect to diameter, but can also be aligned on rotating collectors. ${ }^{[33]}$ Such highly aligned fiber scaffolds can then be cut into monodisperse microcylinders. ${ }^{[34]}$ Importantly, particle diameters are controlled by altering the solution and process parameters during electrohydrodynamic co-jetting, while control over cylinder length is achieved by the microsectioning step. Spatioselective functionalization of one or more compartments of the cylinders has been achieved by incorporation of poly(lactide-co-propargyl glycolide) as an additive during fabrication of the microcylinders, and subsequent modification with biotin and streptavidin by click chemistry. ${ }^{[34]}$ As shown in the Supporting Information, Figure S1, we incorporated a poly[lactide-co- 
(propargyl glycolide)] (acetylene-PLGA) in one of the two compartments. $^{[34]}$

Starting from bicompartmental microcylinders with one functionalized compartment, Scheme 1 depicts how chemically controlled bending is achieved by selective growth of

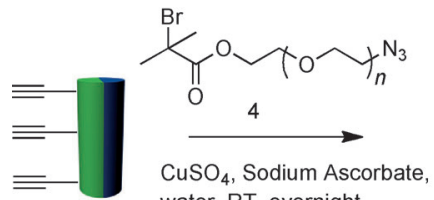

water, RT, overnight

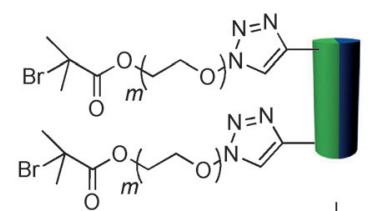

$\mathrm{CuBr}, \mathrm{CuBr}_{2}$, Bipy, OEGMA Water, RT, $1 \mathrm{~h}$<smiles>C=C(CC(C)(Br)C(=O)OCCOCCOC)C(=O)OCCOCCOCCOCCOCCOC</smiles>

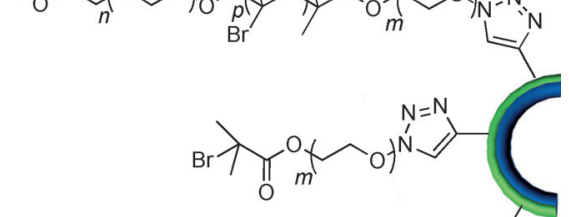<smiles>C=C(C=C(C)C(=O)OCCOn1cc(CC)nn1)C(=O)OCCOCCOC</smiles>

Scheme 1. Selective surface modification of bicompartmental microcylinders with a PEGMA surface layer.

poly(oligo(ethylene glycol) methyl ether methacrylate) (OEGMA, average $M_{\mathrm{n}}$ ca. 475), hydrogel brushes from the surface of the functionalized compartment. The acetylene groups at the surface are converted by $\mathrm{Cu}$-catalyzed Huisgen cycloaddition with the azide-modified ester bromide linker 4. The incorporation of acetylene-PLGA into one compartment allowed for subsequent spatioselective surface modification via copper catalyzed Huisgen heterocycloaddition with an azide functionalized atom-transfer radical polymerization (ATRP) initiator, which was specifically synthesized for this use.

The heterobifunctional linker $\mathbf{4}$ with an azide group as well as an ATRP initiator group was synthesized using a multi-step procedure (Supporting Information, Scheme S1). The overall yield of the synthesis of linker 4 was $70 \%$. Selective monotosylation of PEG diols can be considered the most critical part of the reaction cascade, because a selective reaction is required to ensure a high yield of heterobifunctional PEG. Stefanko et al. ${ }^{[40]}$ reported a synthetic route toward monotosylated PEG 400. In our work, monotosylation of PEG 600 (1) was carried out similarly. As expected, the synthesis of the monotosyl-PEG 600 (2) proceeded with $90 \%$ yield and the different chain length of PEG did not significantly affect the selectivity of the reaction compared to Stefanko's work. Next, the monoazide-PEG 3 was obtained from intermediate $\mathbf{2}$ by nucleophilic substitution of the tosyl groups with azides. The substitution was $100 \%$ and hence, no further purification of compound $\mathbf{3}$ was necessary. The final step to obtain compound $\mathbf{4}$ was the conjugation of 2-bromo isobutyryl bromide to monoazide-PEG in the presence of triethyl amine. The esterification of the hydroxy groups was straightforward and the crude mixture was purified by flash chromatography to obtain compound 4 in good yields (85\%).

To enable controlled bending of the microcylinders, we then immobilized the macroinitiator 4 selectively onto one half of the anisotropic microcylinders using Huisgen 1,3dipolar cycloaddition (Scheme 1). For this purpose, acetylene-PLGA was added to the jetting solution of one compartment (green compartment) and bicompartmental microcylinders with various aspect ratios were prepared. The partially modified microcylinders were reacted with initiator-terminated PEG azide in the presence of copper sulfate and sodium ascorbate. The selective conversion of the acetylene groups on one compartment only required optimization of the reaction conditions. Thus, conversion of the acetylene groups was monitored by infrared spectroscopy. The disappearance of bands for the $\mathrm{CH}$ and $\mathrm{C} \equiv \mathrm{C}$ bond stretches at around 3280 and $2200 \mathrm{~cm}^{-1}$, which can be associated with terminal alkyne groups, was studied as a function of reaction time as well as initiator concentration. After $24 \mathrm{~h}$, the alkyne bands were no longer visible in the spectrum. Therefore, a reaction period of $24 \mathrm{~h}$ was used in subsequent studies. Moreover, the optimum ratio of the reagents was found to be initiator 4: $\mathrm{Cu}^{\mathrm{II}}$ :sodium ascorbate 0.5:0.02:0.04; the concentration of the initiator 4 in aqueous solution was $0.032 \mathrm{M}$. Note that the immobilization of initiator $\mathbf{4}$ by itself did not cause bending of the PLGA microcylinders (Supporting Information, Figure S2).

Before the atom transfer radical polymerization (ATRP) of OEGMA, we washed the cylinders extensively with copious amounts of Tween 20-containing water. This step ensured complete removal of free initiator, which could lead to bulk polymerization in the next step. For surface-initiated ATRP of OEGMA, PLGA microcylinders carrying the macroinitiator 4 on only one hemisphere were dispersed in water. $\mathrm{CuBr}$ along with $10 \% \mathrm{CuBr}_{2}$ and bipyridine were used as the catalyst system. The surface polymerization conditions, especially the concentration of microcylinders had to be optimized. Notably, cylinder concentrations below 30000 cylinders per $\mathrm{mL}$ afforded only heterogeneous mixtures of particles. We attributed this outcome to an unfavorably high concentration of initiator, which may lead to multilayer initiator attachment. ${ }^{[41]}$ We found that about 40000 cylinders per $\mathrm{mL}$ was the optimum microcylinder concentration (Scheme 1). In all cases, polymerization reactions on microcylinders were allowed to proceed for $1 \mathrm{~h}$, which resulted in bending of the cylinders. After the ATRP was complete, the microcylinders were repeatedly washed with $0.1 \mathrm{M}$ EDTA solution to remove excess copper as well as unbound polymer.

Confocal micrographs of bicompartmental microcylinders, (Figure 1), indicate a well-defined surface layer, which is comprised of swollen PEGMA brushes. Moreover, it can be clearly seen that the hydrogel compartment causes the microcylinders to bend outward compared to microcylinders prior to ATRP. We studied how the amount of bending depends on the length of the microcylinders and measured the radii of curvature of the inner $\left(R_{\mathrm{j}}\right)$ and outer $\left(R_{\mathrm{o}}\right)$ surfaces. As shown in Figure $1 \mathrm{f}$, the curvature generally decreases with cylinder length, which agrees with insights from mechanical 
a)

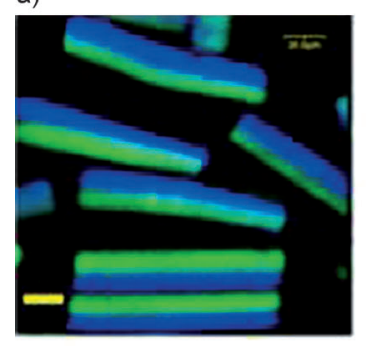

e)

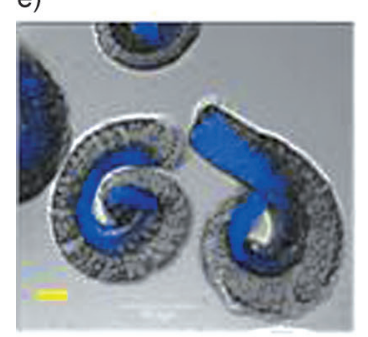

b)

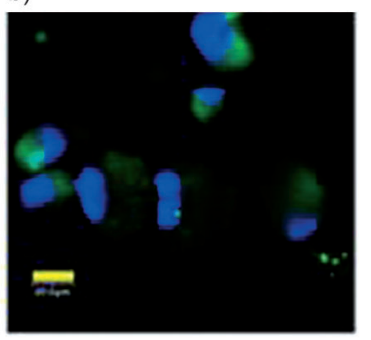

f)

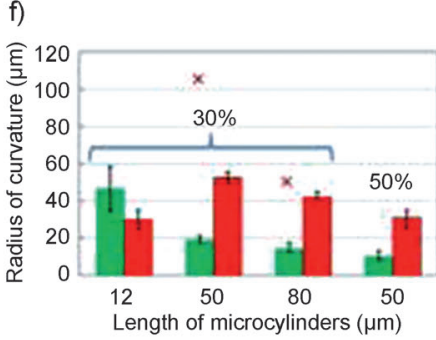

c)

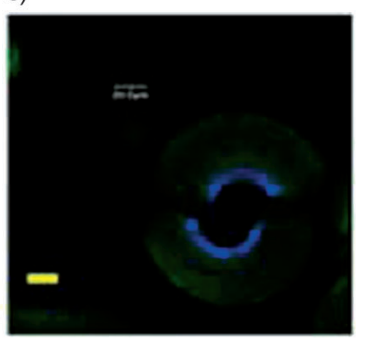

g)

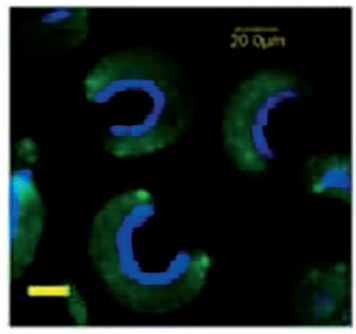

d)

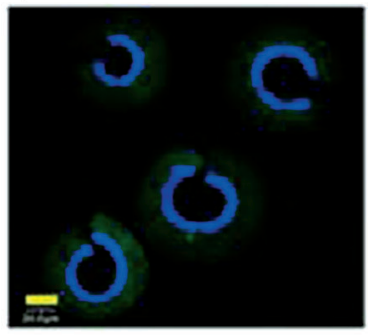

h)

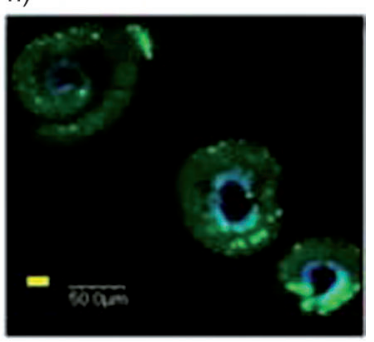

Figure 1. Representative images of a) brush free bicompartmental microcylinders of $120 \mu$ m length (before polymerization) and after spatioselective SIP (surface-initiated polymerization) of OEGMA on microcylinders of different lengths: b) $12 \mu \mathrm{m}, \mathrm{c}) 50 \mu \mathrm{m}, \mathrm{d}) 80 \mu \mathrm{m}, \mathrm{e}) 120 \mu \mathrm{m}$. All multiphasic cylinders contain around $30 \%$ of acetylene-PLGA in one compartment. $f$ ) Measured inner (green) and red (outer) radii of curvature versus length of biphasic microcylinders, for brush bilayers at two different acetylene concentrations (labeled above the data points). Data points $\times$ are from finite element simulations. Effect of grafting density on bending for g) $50 \mu \mathrm{m}$ and h) $80 \mu \mathrm{m}$ biphasic cylinders (after polymerization) which contain $50 \%$ of acetylene-PLGA in one compartment. Yellow scale bars are $20 \mu \mathrm{m}$.

modeling discussed later. Deviations from the trend include short cylinders (Figure 1b), which have high bending stiffness and permit a large amount of the swollen layer to relieve stress by curling around the cylinder ends; and very long cylinders (which cannot be assigned a single radius of curvature) that touch at their ends and sometimes form spirals (Figure $1 \mathrm{e}$ and Figure $1 \mathrm{~h}$ ).

The interchain interactions can give rise to a mechanical stress within the brushes because of the restrictions imposed on the chain ends by the grafting. ${ }^{[42]}$ On a rigid substrate, the chains must stretch vertically to reduce the polymer-polymer interchain repulsion. In contrast, on a flexible substrate, the repulsive interactions can result in bending of the substrate. This resultant in-plane stress causes the microcylinders to bend. Because the bending stiffness of the cylinders depends on their length and diameter, as well as the mechanical properties of the compartments, the curvature can be tuned by the initial cylinder geometry and composition. Moreover, for hydrogel graft-layers, the initiator density on the surface has pronounced influences on the architecture of the hydrogel. We thus investigated two different graft densities, which were simply varied by adjusting the concentration of the acetylene-modified PLGA (30\% versus $50 \%$ ) in one of the jetting solutions. Figure $1 \mathrm{~g}$ and $\mathrm{h}$ show distinct particle architectures for two different cylinder lengths at 50\% compared to $30 \%$ acetylene-PLGA (Figure 1c and d). For both lengths, the higher graft density results in a smaller radius of curvature.

Based on several SEM and confocal images (Figure 2), the swelling ratio of the microcylinders (measured by dividing the diameter of the swollen cylinder by the diameter of the dry cylinder) can be estimated to be $1.64 \pm 0.2$, which is com-
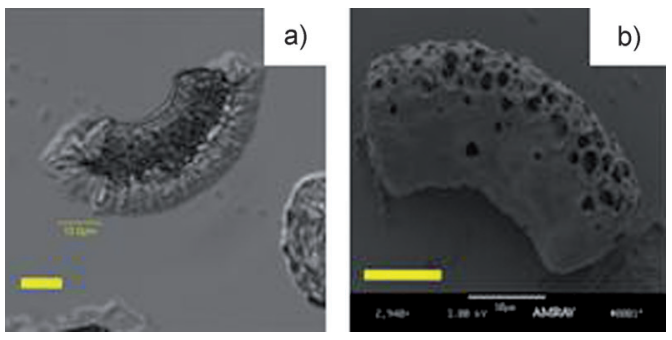

Figure 2. Representative images of PEGMA brushes on bicompartmental microcylinders of $30 \mu \mathrm{m}$ length: a) in water (confocal image); b) in dry state (SEM image). Yellow scale bars: $10 \mu \mathrm{m}$. All biphasic cylinders contain $30 \%$ of acetylene-PLGA in one compartment.

parable to swelling ratios found in gold/polymer hybrid materials. ${ }^{[43]}$ After lyophilization, water was removed from hydrogel portion of the cylinders, which took on a semiporous architecture. Incidentally, the curvature caused by growth of the PEGMA layer is comparable to that previously achieved in free-standing Au-brush bilayer films. ${ }^{[44]}$

The observed relationships between geometry, graft density, and bending have also been investigated computationally by a 3D large deflection finite element analysis (FEA). In this model, the surface stress was represented as the swelling \% of the brush layer. The finite element model consists of a semicylinder (Young's Modulus $E_{1}$, see Figure 3), partially intersected with a cylinder $\left(E_{2}\right)$. The region of the semicylinder that did not intersect with the cylinder represented the swelling outer compartment, which was rigidly coupled to the cylinder. Swelling (S) was modeled as isotropic thermal expansion. The final swollen geometry was used to 
calculate the radius of curvature by fitting a circle through the endpoints and midpoint of the inner surface of the microcylinder (i.e., the surface with negative curvature). Video S1 (Supporting Information) shows the simulated actuation of a cylinder in half-symmetry with initial length $L=80 \mu \mathrm{m}$, diameter $D=15 \mu \mathrm{m}$, initial PEGMA (outer compartment) thickness $t=3 \mu \mathrm{m}$ and $S=50 \%$.

To accurately simulate the final geometry, the elastic properties of the constituent polymers PLGA and PEGMA are required. However, the mechanical stiffness of anisotropic semi-crystalline PLGA microfibers depends on the fiber diameter; for example, fibers with $4 \mu \mathrm{m}$ diameter were found to have $E=205 \mathrm{MPa}$ along the fiber ${ }^{[45]}$ To capture this effect, the influence of the modulus ratio $\left(E_{2} / E_{1}\right)$ on the actuation of the bicompartmental PLGA/PEGMA microcylinders was modeled. Simulations were carried out up to $S=65 \%$, and beyond this point, convergence was prevented by excessive deformation of the elements at the ends of the cylinder. Nevertheless, based on the theory of elasticity for differential thermal expansion, ${ }^{[46]}$ we used the simulation results to establish a family of power-law curves that predict the actuation behavior. Specifically, in Figure 3, we have plotted the relationships between the predicted radius of curvature and the modulus ratio, the initial cylinder length, and the amount of swelling.

The simulations emphasize how the bicompartmental cylinder design can achieve highly tunable chemically driven actuation. We also find that the simulations accurately predict the observed trend of decreasing curvature with increasing cylinder length. For example, actuation of a cylinder with $L=$ $80 \mu \mathrm{m}$ at $S=380 \%$ corresponds to a radius of curvature of
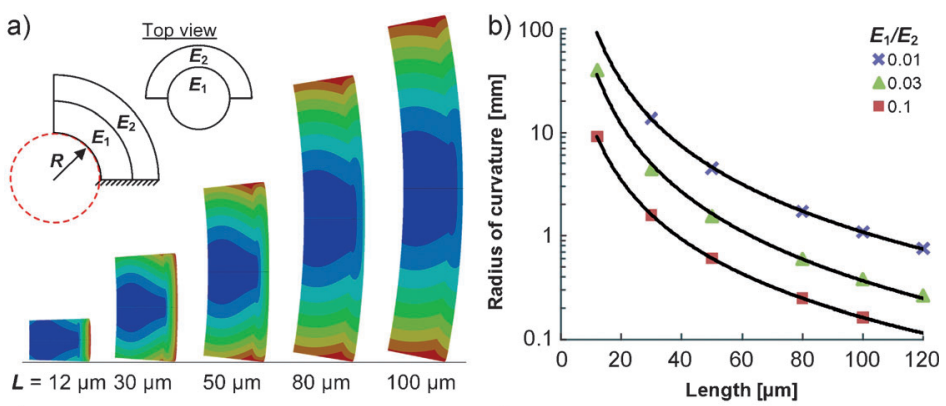

c)
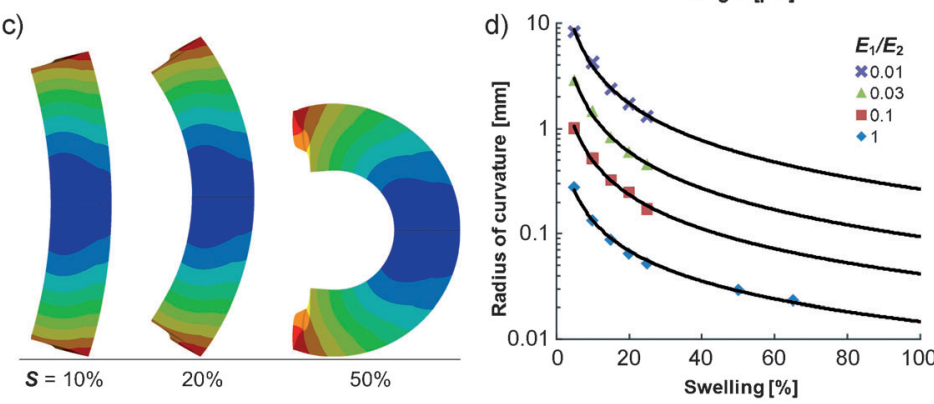

Figure 3. Large deflection finite element simulations of microcylinder actuation: a) final predicted geometry of cylinders with varying length, for $20 \%$ swelling of the outer compartment; b) curvature trends versus length $(S=20 \%)$, for different elastic modulus ratios; c) predicted geometry of cylinders with the same initial length $(L=80 \mu \mathrm{m})$ and varying amounts of swelling; d) curvature trends versus amount of swelling, for different modulus ratios. In all simulations, $D=15 \mu \mathrm{m}$ and $t=3 \mu \mathrm{m}$.
$R=57 \mu \mathrm{m}$ in our simulation, while a microcylinder with $L=$ $50 \mu \mathrm{m}$ yields $R=110 \mu \mathrm{m}$. These are both greater than measured values in Figure 1, but are within reason considering uncertainties in the mechanical properties of the cylinders, the assumption of constant surface stress, and the estimation of the amount of volume change based on the measured diameters in the swollen and dry states.

Moving beyond Janus microcylinders, one of the major differences of the electrohydrodynamic co-jetting approach over conventional methods, such as macroscopic gold stripes from free-standing films, ${ }^{[4]}$ is that the internal architecture of the composite materials, and thus the mechanical responses, can be tuned in controlled ways. Simple alteration of the parallel flows that are used during electrohydrodynamic cojetting can result in a wide range of different microcylinder architectures, which can yield controllable mechanical responses. To highlight this aspect, tetracompartmental microcylinders (Figure 4) were generated by loading diagonally opposing nozzles in a square configuration with blue and green dyes. ${ }^{[3]}$ Two different types of tetracompartmental microcylinders were produced: one with green dye in one quarter and blue dye in the remaining three quarters (Figure $4 \mathrm{~d}$ ) and another one with a cross configuration, where green dye was loaded in opposing quarters and blue dye in the two remaining compartments (Figure 4b). The different dyes signify compartments loaded with either neat PLGA (blue) or acetylene-PLGA (green), which is the component that will give rise to the hydrogel coating following the preparative approach outlined in Scheme 1.

In these examples, approximately $30 \%$ of acetylenePLGA was loaded into the jetting solution that led to the green compartments. $80 \mu \mathrm{m}$ long microcylinders were prepared, as described above. Subsequent immobilization of the initiator 4 followed by ATRP of PEGMA resulted in the introduction of polymer brush only into the green quarters, while the blue compartments remained unchanged. Distinctly different bending transitions were observed among these different types of tetracompartmental microcylinders. When two opposite faces have equally thick swollen brushes, the mechanical stresses within the brush layer nullify each other and result in a butterfly shape, where the microcylinder remains straight. This situation is distinctly different from the semicircular bending that was observed, when only one quarter is surfacemodified with PEGMA. In the latter case, the extent of bending was however less pronounced than for their bicompartmental analogues, where the PEGMA compartment made up $50 \%$ of the cylinders (compare Figure $4 \mathrm{e}$ and Figure 1d). Moreover, inhomogenities in the compartmentalization give rise to instabilities that result in non-uniform bending and sometimes twisting of the microcylinders in Figure 4c (see red arrows). The different bending behavior of the microcylinders shown in Figure 4 underpins the importance of the internal positioning of the hydrogel compartment relative to the non-swelling PLGA. Notably, the target architecture as well as the degree of bending can be easily tuned in our approach by adjusting the 


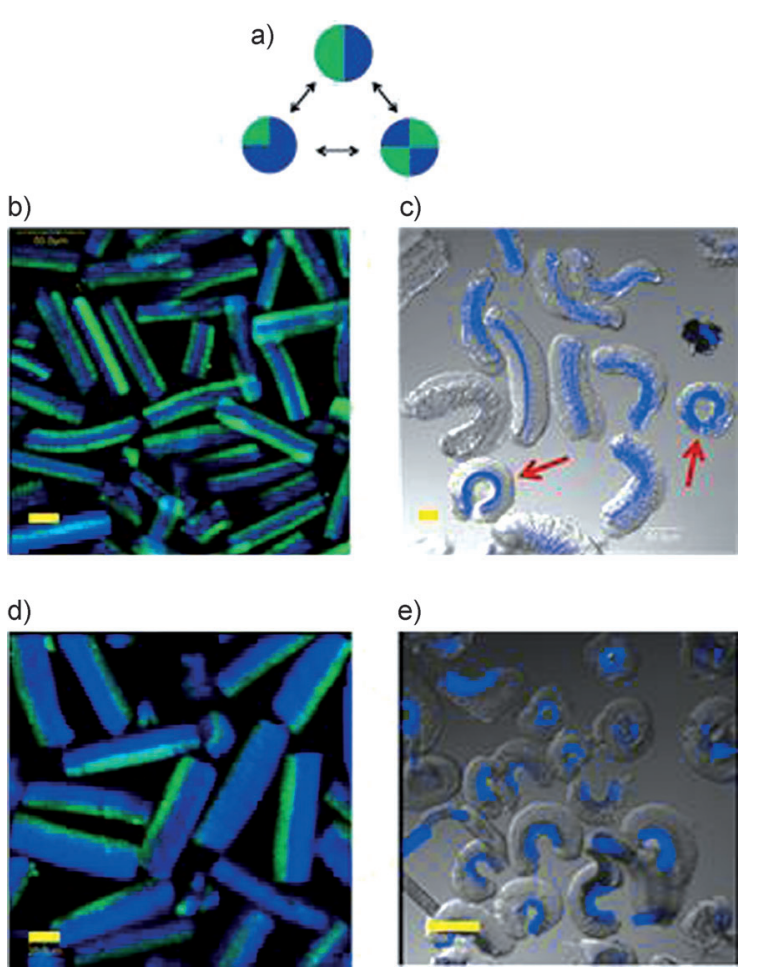

Figure 4. a) Complex compartmentalization based on electrohydrodynamic co-jetting in different fluid configurations. Representative images of tetracompartmental microcylinders having PEGMA brushes in b) two opposite quarters, before polymerization; c) two opposite quarters, after polymerization; red arrows indicate twisting, d) one quarter , before polymerization; and e) one quarter, after polymerization. Yellow scale bars: $20 \mu \mathrm{m}$ except for (d): $100 \mu \mathrm{m}$. All tetracompartmental $80 \mu \mathrm{m}$ cylinders contain $30 \%$ of acetylene-PLGA in the green compartments.

processing conditions during electrohydrodynamic co-jetting.

Based on electrohydrodynamic co-jetting and spatioselective surface modification, we have developed a new type of active polymer microcylinders. The cylinders are comprised of a water-swellable hydrogel compartment as well as an inert compartment and thus undergo substantial bending in water. Predictable fine-tuning of the microcylinders ranging from simple bent to coiled structures can be achieved by adjusting the aspect ratio of the microcylinders. A wide range of water-soluble polymers can be selectively grown using this strategy, which will allow for further adjustment of the morphology and responsive behavior of the microcylinders. Future work will thus need to continue to diversify the types of materials that can be incorporated into the different compartments. Careful selection of intrinsically dissimilar polymers may lead to additional attributes, such as reversibility or the ability to switch between convex and concave structures. Conceptually, this work may establish a new route towards highly miniaturized transducer particles that, with further work, may find applications as biosensors, shape-memory devices, or microactuators. ${ }^{[47]}$

Received: July 30, 2011

Revised: October 12, 2011

Published online: December 7, 2011
Keywords: anisotropic materials - bent molecules . click chemistry $\cdot$ microcylinders $\cdot$ polymerization

[1] R. Messing, A. M. Schmidt, Polym. Chem. 2011, 2, 18.

[2] Y. Osada, J. P. Gong, Adv. Mater. 1998, 10, 827.

[3] "Hydrogel Sensors and Actuators": A. Richter, Springer Ser. Chem. Sens. Biosens. 2009, 6, 221.

[4] Y. Hu, W. Chen, Macromol. Chem. Phys. 2011, 212, 992.

[5] C. Amiri Naini, S. Franzka, S. Frost, M. Ulbricht, N. Hartmann, Angew. Chem. 2011, 123, 4606; Angew. Chem. Int. Ed. 2011, 50, 4513.

[6] H. Yoon, J. H. Ahn, P. W. Barone, K. Yum, R. Sharma, A. A. Boghossian, J. H. Han, M. S. Strano, Angew. Chem. 2011, 123, 1868; Angew. Chem. Int. Ed. 2011, 50, 1828.

[7] Z. Zhang, E. M. Olsen, M. Kryger, N. V. Voigt, T. Torring, E. Gueltekin, M. Nielsen, R. Mohammad Zadegan, E. S. Andersen, M. Nielsen, Angew. Chem. 2011, 123, 4069; Angew. Chem. Int. Ed. 2011, 50, 3983.

[8] "Dielectrics for Nanosystems 4": B. L. Gray, ECS Trans. 2010, $28,535$.

[9] J. Bünsow, T. S. Kelby, W. T. S. Huck, Acc. Chem. Res. 2010, 43, 466.

[10] H. Yu, T. Ikeda, Adv. Mater. 2011, 23, 2149.

[11] A. Lendlein, R. Langer, Science 2002, 296, 1673.

[12] W. Small IV, P. Singhal, T. S. Wilson, D. J. Maitland, J. Mater. Chem. 2010, 20, 3356.

[13] Z. Chen, X. Tan, Sens. Actuators A 2010, 157, 246.

[14] M. E. Piyasena, R. Newby, T. J. Miller, B. Shapiro, E. Smela, Sens. Actuators B 2009, 141, 263.

[15] T. Xie, Nature 2010, 464, 267.

[16] E. Smela, Biomed. Appl. Electroact. Polym. Actuators 2009, 249.

[17] Y. Zou, A. Lam, D. E. Brooks, A. Srikantha Phani, J. N. Kizhakkedathu, Angew. Chem. 2011, 123, 5222; Angew. Chem. Int. Ed. 2011, 50, 5116.

[18] D. Dendukuri, D. C. Pregibon, J. Collins, T. A. Hatton, P. S. Doyle, Nat. Mater. 2006, 5, 365.

[19] J. P. Rolland, B. W. Maynor, L. E. Euliss, A. E. Exner, G. M. Denison, J. M. DeSimone, J. Am. Chem. Soc. 2005, 127, 10096.

[20] A. Walther, X. André, M. Drechsler, V. Abetz, A. H. Müller, J. Am. Chem. Soc. 2007, 129, 6187.

[21] H. Y. Chen, J. M. Rouillard, E. Gulari, J. Lahann, Proc. Natl. Acad. Sci. USA 2007, 104, 11173.

[22] Y. Y. Yu, S. S. Chang, C. L. Lee, C. R. C. Wang, J. Phys. Chem. B 1997, 101, 6661.

[23] K. Esumi, K. Matsuhisa, K. Torigoe, Langmuir 1995, 11, 3285.

[24] V. M. Cepak, C. R. Martin, J. Phys. Chem. B 1998, 102, 9985.

[25] B. R. Martin, D. J. Dermody, B. D. Reiss, M. M. Fang, L. A. Lyon, M. J. Nathan, T. E. Mallouk, Adv. Mater. 1999, 11, 1021.

[26] S. Fullam, D. Cottel, H. Rensmo, D. Fitzmauritce, Adv. Mater. 2000, 12, 1430.

[27] N. R. Jana, L. Gearheart, C. J. Murphy, Adv. Mater. 2001, 13, 1389.

[28] J. W. Kim, R. J. Larsen, D. A. Weitz, J. Am. Chem. Soc. 2006, 128, 14374.

[29] J. W. Kim, R. J. Larsen, D. A. Weitz, Adv. Mater. 2007, 19, 2005.

[30] T. Tanaka, M. Okayama, Y. Kitayama, Y. Kagawa, M. Okubo, Langmuir 2010, 26, 7843.

[31] H. Yu, M. Chen, P. M. Rice, S. X. Wang, R. L. White, S. Sun, Nano Lett. 2005, 5, 379.

[32] K. H. Roh, D. C. Martin, J. Lahann, Nat. Mater. 2005, 4, 759.

[33] S. Bhaskar, J. Lahann, J. Am. Chem. Soc. 2009, 131, 6650.

[34] S. Bhaskar, J. Hitt, S. L. Chang, J. Lahann, Angew. Chem. 2009, 121, 4659; Angew. Chem. Int. Ed. 2009, 48, 4589.

[35] S. Bhaskar, K. H. Roh, X. Jiang, G. L. Baker, J. Lahann, Macromol. Rapid Commun. 2008, 29, 1655. 
[36] S. Mandal, S. Bhaskar, J. Lahann, Macromol. Rapid Commun. 2009, 30, 1638.

[37] K. J. Lee, J. Yoon, J. Lahann, Curr. Opin. Colloid Interface Sci. 2011, 16, 195.

[38] K. H. Roh, M. Yoshida, J. Lahann, Langmuir 2007, 23, 5683.

[39] a) S. Bhaskar, K. M. Pollock, M. Yoshida, J. Lahann, Small 2010, 6, 404; b) S. Bhaskar, C. T. Gibson, M. Yoshida, H. Nandivada, X. Deng, N. H. Voelcker, J. Lahann, Small 2011, 7, 812.

[40] M. J. Stefanko, Y. K. Gun'ko, D. K. Rai, P. Evans, Tetrahedron 2008, 64, 10132

[41] J. Iruthayaraj, S. Chernyy, M. Lillethorup, M. Ceccato, T. Røn, M. Hinge, P. Kingshott, F. Besenbacher, S. U. Pedersen, K Daasbjerg, Langmuir 2011, 27, 1070.
[42] Y. Lyatskaya, A. C. Balazs, Macromolecules 1996, 29, 5469.

[43] N. Cheng, O. Azzaroni, S. Moya, W. T. S. Huck, Macromol. Rapid Commun. 2006, 27, 1632.

[44] T. S. Kelby, W. T. S. Huck, Macromolecules 2010, 43, 5382.

[45] V. Milleret, B. Simona, P. Neuenschwander, H. Hall, Eur. Cells Mater. 2011, 21, 286.

[46] S. Timoshenko, J. Opt. Soc. Am. Rev. Sci. Instrum. 1925, 11, 233.

[47] S. A. Wilson, R. P. J. Jourdain, Q. Zhang, R. A. Dorey, C. R. Bowen, M. Willander, Q. U. Wahab, M. Willander, S. M. Al-hilli, O. Nur, E. Quandt, C. Johansson, E. Pagounis, M. Kohl, J. Matovic, B. Samel, W. Wijngaart, E. W. H. Jager, D. Carlsson, Z. Djinovic, M. Wegener, C. Moldovan, R. Iosub, E. Abad, M. Wendlandt, C. Rusu, K. Persson, Mater. Sci. Eng. 2007, R56, 1. 